\title{
Development of an acoustic diagnostic for determination of heat release rate perturbations in pulsated flames
}

\author{
Jingxuan Li ${ }^{\mathrm{a}}$, Franck Richecoeur And ThierRy Schuller \\ Laboratoire EM2C, CNRS et École Centrale Paris, 92295 Châtenay-Malabry, France
}

Received 23 April 2011, Accepted 1 June 2011

\begin{abstract}
Heat release rate fluctuations are the source of combustion instabilities that cause recurrent problems in many combustors. These quantities are difficult to measure and optical techniques are generally implemented. The initial validation of an alternative method is presented in the case of pulsated laminar premixed flames. The technique relies on determining the travel time of ultrasonic waves propagating through the flow. The link between heat release rate fluctuations and perturbations in the travel time of ultrasonic waves is established. Measurements made with this method are compared with optical measurements and an analytical model. A good agreement is obtained for the gain and phase of the transfer function between perturbations in the sound travel time and heat release rate disturbances when the flame is submitted to harmonic modulations of the flow. This work validates the principle of the proposed technique and lays the foundations for future developments.
\end{abstract}

Key words: Heat release rate / ultrasonic waves / premixed flames / sound travel time

\section{Introduction}

Heat release rate disturbances in combustion chambers are often responsible for increased heat fluxes to the walls, vibration and noise that can eventually degenerate into thermo-acoustic instabilities and cause early aging of some components of the combustion chamber, or even greater equipment damages in worse cases [1]. These disturbances are difficult to measure in the combustion chamber because there is no diagnostic that allows direct access to heat release rate.

Optical methods are generally implemented at the laboratory. They are based on recording the chemiluminescence emission from the flame or the use of more complex diagnostics based on laser-induced fluorescence. These latter techniques are cumbersome to implement in practical configurations and are still in development. Moreover, time resolved measurements are limited by the power and speed of the lasers and cameras $[2,3]$. The use of the chemiluminescence emission signal from the flame remains the preferred tool for analyzing disturbances in heat release rate. Natural excited radicals, like $\mathrm{OH}^{*}, \mathrm{CH}^{*}$ or $\mathrm{C}_{2}{ }^{*}$, are generally considered as good markers of heat release rate $[4,5]$. A direct interpretation of the chemiluminescence signal is restricted to well premixed flames when the mixture composition is uniform. Analysis of this

\footnotetext{
${ }^{a}$ Corresponding author: Jingxuan.1i@em2c.ecp.fr
}

signal for partially premixed or non-premixed configurations, effects of flame stretching, flame curvature, turbulent intensity, pressure and gases composition remain a field of active investigation [6-8]. It is therefore interesting to explore alternative diagnostics when optical access to the combustion chamber is limited.

The objective of this study is to develop a method based on the exploitation of the propagation time of ultrasonic waves traveling through the flow to access to heat release rate disturbances in perturbed flames. The use of ultrasonic waves to probe the flow is widespread, however, it has not been considered so far to access to heat release rate. These waves offer attractive features since they barely disturb the combustion dynamics [9]. These waves are scattered by turbulent flames which can potentially reduce the SNR of the proposed technique [10].

The principle of the method is presented in Section 2. A theoretical link is established between fluctuations on heat release rate and travel time of ultrasonic waves passing through laminar premixed flames submitted to harmonic modulations of the upstream flow. The experimental setup used to validate the predictions is described in Section 3. Optical and acoustic measurements of heat release rate perturbations are compared with theoretical predictions in Section 4. These elements are used to determine the sensitivity of the acoustic method and it is demonstrated that it is possible to probe reacting flows with ultrasonic waves. 


\section{Nomenclature}

\begin{tabular}{|c|c|}
\hline$A$ & Flame surface area $\left[\mathrm{m}^{2}\right]$ \\
\hline$B$ & Chirp frequency bandwidth $[\mathrm{Hz}]$ \\
\hline$c$ & Speed of sound $\left[\mathrm{m} \cdot \mathrm{s}^{-1}\right]$ \\
\hline$f_{\mathrm{i}}$ & Initial frequency of chirp signal $[\mathrm{Hz}]$ \\
\hline$f$ & Modulation frequency $[\mathrm{Hz}]$ \\
\hline$H$ & Flame height $[\mathrm{m}]$ \\
\hline $\mathcal{H}$ & Transfer function between heat release rate and sound travel time [-] \\
\hline$I$ & Light-emission intensity $[\mathrm{V}]$ \\
\hline$k$ & Convective wave number $\left[\mathrm{m}^{-1}\right]$ \\
\hline$L$ & Length of acoustic path $[\mathrm{m}]$ \\
\hline$L_{f}$ & Characteristic length of burned gases $[\mathrm{m}]$ \\
\hline$\dot{m}$ & Mass flow rate $\left[\mathrm{kg} \cdot \mathrm{s}^{-1}\right]$ \\
\hline$\dot{Q}$ & Heat release rate $[\mathrm{W}]$ \\
\hline$r_{i}$ & Local flame front radius $[\mathrm{m}]$ \\
\hline$r_{o}$ & Local position of the interface between burned gases and ambient air [m] \\
\hline$P M$ & Photomultiplier \\
\hline$R$ & Burner nozzle outlet radius $[\mathrm{m}]$ \\
\hline$S N R$ & Signal to noise ratio $[-]$ \\
\hline$S_{\mathrm{L}}$ & Laminar burning velocity $\left[\mathrm{m} . \mathrm{s}^{-1}\right]$ \\
\hline$t_{\mathrm{r}}$ & Repetition time of the chirp signal $[\mathrm{s}]$ \\
\hline$t$ & Time $[s]$ \\
\hline$v$ & Flow velocity $\left[\mathrm{m} \cdot \mathrm{s}^{-1}\right]$ \\
\hline$\Delta t$ & Sound propagation time $[\mathrm{s}]$ \\
\hline$\alpha$ & Half angle of flame cone [rad] \\
\hline$\beta$ & Amplitude of the chirp signal $[-]$ \\
\hline$\Gamma$ & Dimensionless flame parameter [-] \\
\hline$\rho$ & Gas density $\left[\mathrm{kg} \cdot \mathrm{m}^{-3}\right]$ \\
\hline$\phi$ & Mixture equivalence ratio $[-]$ \\
\hline$\omega$ & Angular frequency $\left[\mathrm{s}^{-1}\right]$ \\
\hline$\omega^{*}$ & Reduced angular frequency $[-]$ \\
\hline$\tau$ & Time duration of chirp signal $[\mathrm{s}]$ \\
\hline \multicolumn{2}{|r|}{ Subscripts and superscripts } \\
\hline $\mathrm{b}$ & Burned gases \\
\hline $\mathrm{u}$ & Unburned gases \\
\hline rms & Root mean square value \\
\hline- & Mean component \\
\hline & Fluctuating component \\
\hline
\end{tabular}

\section{Model of the sound travel time and heat release rate}

The analysis is restricted to laminar premixed flame submitted to flow velocity modulations at a fixed equivalence ratio. The wavelength of flow disturbances is considered large enough compared to the flame thickness to consider the flame front as a thin interface separating the reactive mixture from the combustion products. The flame front propagates normal to itself at the laminar burning velocity $S_{\mathrm{L}}$, stretch and curvature effects are ignored in the study. Successive approximations are made to obtain an analytical expression of heat release rate perturbations versus sound travel time disturbances. Some assumptions are relatively crude, but these developments offer a simple theoretical framework to test the effects of different parameters controlling the response of the sound travel time and validate the proposed technique. This analysis is then supplemented by an independent

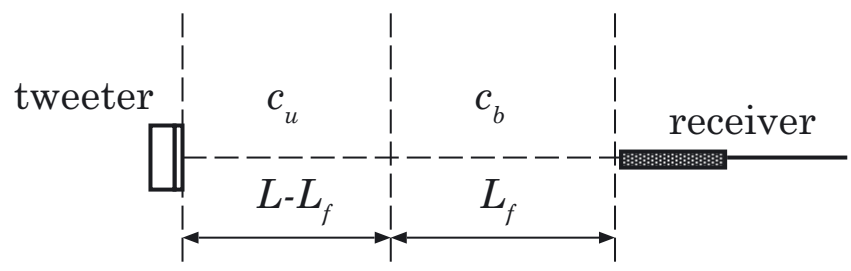

Fig. 1. Schematic view of two different homogenous gases characterized by different densities and speed of sound between the transmitter and receiver.

experimental validation based on a classical optical technique.

It is worth starting with the case of two homogeneous gases comprised between a transmitter and a receiver. This situation is illustrated in Figure 1. Ultrasonic waves propagate through the fresh gases mixture with a speed of 
sound of $c_{u}$ over a distance of $L-L_{f}$ and the burned gases over a distance of $L_{f}$ characterized by a speed of sound $c_{b}$. It is supposed that the flame is an interface separating the two media by a jump in density and speed of sound. In this situation, the sound travel time $\Delta t$ between the transmitter and receiver separated by a distance $L$ can be written:

$$
\Delta t=\frac{L-L_{f}}{c_{u}}+\frac{L_{f}}{c_{b}}=\Delta t_{0}-\frac{L_{f}}{c *}
$$

where $\Delta t_{0}$ is the travel time of ultrasonic waves crossing the distance $L$ when there is only fresh mixture between the transmitter and receiver and $c *=c_{b} c_{u} /\left(c_{b}-c_{u}\right)$ is an equivalent speed of sound. Low frequency disturbances in heat release rate are now considered with the condition that the flow modulation frequency remains low compared to the inverse of sound propagation time: $f \Delta t \ll 1$. In these conditions, ultrasonic waves cross the disturbed flame in a frozen flow. Heat release rate perturbations originate from undulations of the flame front that wrinkles when submitted to the flow velocity modulation [1]. Disturbances of the position of the flame front $L_{f}$ indirectly induce changes in sound travel time:

$$
\Delta t^{\prime}=-\frac{L_{f}^{\prime}}{c *}
$$

In this expression only disturbances in the acoustic path length $L_{f}$ are taken into account, while fluctuations in the temperature and composition of the gases in the fresh and hot gases are neglected. This approximation is valid as long as relative fluctuations of the speed of sound remain small $\left(c *^{\prime} / \overline{c *} \ll L_{f}^{\prime} / \overline{L_{f}}\right)$. Consider now the case of a conical premixed flame stabilized on an axisymmetric burner presented in Figure 4. This generic configuration is used to validate the proposed technique. In this situation, ultrasonic waves cross ambient air and the burned gases characterized by a speed of sound $c_{b}$ separated from the fresh reactants characterized by a speed of sound $c_{u}$. To simplify the problem, the fresh gases and ambient air are characterized by the same density and speed of sound $c_{0}=c_{u}$. This is a reasonable approximation for reactants injected at the ambient air temperature and which are strongly diluted by nitrogen. In this case, the length of acoustic path in the burned gases is modified to $L_{f}=2\left(r_{o}-r_{i}\right)$, where $r_{o}$ represents the radius of the interface between the hot plume and fresh ambient air, which moves when the position $r_{i}$ of the flame front is perturbed (see Fig. 2).

Considering the elementary control volume represented by the gray zone in Figure 2 with a thickness $\mathrm{d} y$ along the axial direction, a mass balance gives a relationship between the radius $r_{i}$ of the flame front and the burned gases $r_{o}$ at time $t$. The instantaneous quantities $a(t)$ are decomposed into their average over a period and a disturbance : $a(t)=\bar{a}+a^{\prime}$ where $a^{\prime} / \bar{a} \ll 1$. The link between the steady-state variables can be written as:

$$
\bar{\rho}_{\mathrm{b}} \bar{v}_{\mathrm{b}}\left(\bar{r}_{o}^{2}-\bar{r}_{i}^{2}\right)-\bar{\rho}_{\mathrm{u}} \bar{v}_{\mathrm{u}}\left(R^{2}-\bar{r}_{i}^{2}\right)=0
$$

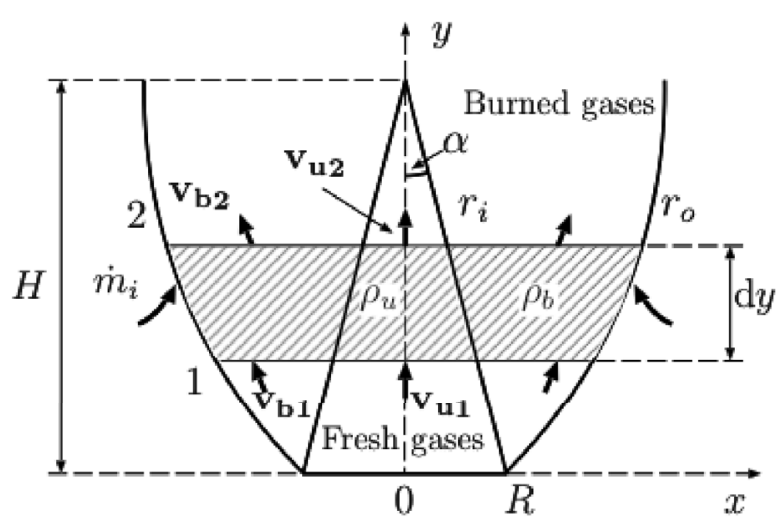

Fig. 2. Schematic representation of a laminar conical premixed flame surrounded by burned gases and ambient air. $H$ indicates the flame height and $\alpha$ half the angle of the flame cone.

In this expression, the lateral surface of the shadow zone in contact with ambient air is supposed to be impermeable $\left(\dot{m}_{i}=0\right)$. The velocities $v_{u}$ and $v_{b}$ correspond to the axial components of the velocity field $\mathbf{v}$ in the fresh mixture and burned gases. Considering low frequency wrinkles along the flame front with a wavelength large compared to the thickness $\mathrm{d} y$, a first-order perturbation analysis of Equation (3) yields the link between fluctuations in the position of the flame front $r_{i}^{\prime}$ and that of the burned gases $r_{o}^{\prime}$ :

$$
\bar{\rho}_{\mathrm{b}} \bar{v}_{\mathrm{b}}\left(\bar{r}_{o} r_{o}^{\prime}-\bar{r}_{i} r_{i}^{\prime}\right)+\bar{\rho}_{\mathrm{u}} \bar{v}_{\mathrm{u}} \bar{r}_{i} r_{i}^{\prime}=0
$$

This expression is valid as long as the velocity fluctuations remain small compared to the flow velocity $v^{\prime} / \bar{v} \ll 1$ and density fluctuations in fresh mixture and burned gases can be neglected. The relationship between the velocity in fresh mixture and burned gases for a conical flame inclined at an angle $\alpha$ is known $\bar{v}_{\mathrm{b}} / \bar{v}_{\mathrm{u}}=$ $1+\left(\bar{\rho}_{\mathrm{u}}-\bar{\rho}_{\mathrm{b}}\right) / \bar{\rho}_{\mathrm{b}} \sin ^{2} \alpha$ [11]. It is then possible to express the relationship between the positions:

$$
\bar{r}_{o}^{2}=\Gamma \bar{r}_{i}^{2}+(1-\Gamma) R^{2} \text { and } r_{o}^{\prime}=\Gamma \frac{\bar{r}_{i}}{\bar{r}_{o}} r_{i}^{\prime}
$$

where the dimensionless quantity $\Gamma=1-\left(\bar{\rho}_{\mathrm{u}} / \bar{\rho}_{\mathrm{b}}\right)[1+$ $\left.\left(\bar{\rho}_{\mathrm{u}}-\bar{\rho}_{\mathrm{b}}\right) / \bar{\rho}_{\mathrm{b}} \sin ^{2} \alpha\right]^{-1}$ is fixed for given flow operating conditions.

It is now necessary to have a model to describe the evolution of the flame front displacement as a function of the imposed velocity modulation. The solution of this problem has been examined in numerous studies [12-14]. This takes the form of a transport equation for the perturbed flame front position $r_{i}^{\prime}$ which is convected along the flame front by the mean flow velocity parallel to the flame front and generated by the normal component of the velocity perturbation to the flame front. This writes:

$$
\frac{\partial r_{i}^{\prime}}{\partial t}+\bar{v}_{\mathrm{u}} \cos ^{2} \alpha \frac{\partial r_{i}^{\prime}}{\partial y}=v_{\mathrm{u}}{ }^{\prime} \tan \alpha
$$


Flow perturbations generated at the burner outlet are transported by the mean flow and can be well described by a convective wave along the axial direction $v_{\mathrm{u}}{ }^{\prime}(y, t)=$ $\tilde{v}_{\mathrm{u}} \exp (i k y-i \omega t)$ when the flow is modulated harmonically [15]. In this expression the convective wave number $k=\omega / \bar{v}_{\mathrm{u}}$ is based on the mean value of flow velocity $\bar{v}_{\mathrm{u}}$ and $\omega=2 \pi f$ is the angular frequency of modulation. The response of the flame front displacement $r_{i}$ is also harmonic and takes the form $r_{i}(y, t)=\tilde{r}_{i}(y) \exp (-i \omega t)$. By injecting these expressions to the equation above, one finds:

$$
\tilde{r}_{i}(y)=\frac{\tilde{v}_{\mathrm{u}}}{\bar{v}_{\mathrm{u}}} \frac{1}{i k \sin \alpha \cos \alpha}\left[\exp \left(\frac{i k y}{\cos ^{2} \alpha}\right)-\exp (i k y)\right]
$$

The heat release rate is given by $\dot{Q}=\int \rho_{\mathrm{u}} S_{L} \Delta q \mathrm{~d} A$, where $\Delta q$ is the heat release rate per unit mass of mixture. For well premixed flames at a fixed equivalence ratio, perturbations in the heat release rate are only function of flame surface area disturbances $A^{\prime}=\tilde{A} \exp (-i \omega t)$. By integrating the above expression along the flame front and neglecting the motion at the base of the flame, it finally comes [13]:

$$
\begin{aligned}
\frac{\tilde{\dot{Q}}}{\overline{\dot{Q}}}=\frac{\tilde{A}}{\bar{A}}= & \frac{\tilde{v}_{\mathrm{u}}}{\bar{v}_{\mathrm{u}}} \frac{2}{\omega_{*}^{2}} \frac{1}{1-\cos ^{2} \alpha} \\
& \times\left[1-\exp \left(i \omega_{*}\right)+\frac{\exp \left(i \omega_{*} \cos ^{2} \alpha\right)-1}{\cos ^{2} \alpha}\right]
\end{aligned}
$$

Perturbations in the positions of the flame front and the interface between the burned gases and ambient air correspond also to time harmonic signals which induce harmonic disturbances in the sound travel time of ultrasonic waves crossing this flow $\Delta t^{\prime}=\widetilde{\Delta t} \exp (-i \omega t)$, where:

$$
\frac{\widetilde{\Delta t}}{\overline{\Delta t}-\overline{\Delta t}_{0}}=\frac{\tilde{r}_{o}-\tilde{r}_{i}}{\bar{r}_{o}-\bar{r}_{i}}
$$

By placing the transmitter and the receiver at half height of the flame $y=H / 2=R /(2 \tan \alpha)$ and combining Equations (5), (7), (8) and (9), one obtains the link between perturbations in heat release rate and sound travel time:

$$
\begin{aligned}
\mathcal{H} & =\frac{\widetilde{\dot{Q}} / \overline{\dot{Q}}}{\widetilde{\Delta t} /\left(\Delta t_{0}-\overline{\Delta t}\right)}=\frac{(4-3 \Gamma)^{1 / 2}-1}{\Gamma(4-3 \Gamma)^{-1 / 2}-1} \\
& \times \frac{i}{\omega_{*}} \frac{\left[1-\exp \left(i \omega_{*} \cos ^{2} \alpha\right)\right]-\left[1-\exp \left(i \omega_{*}\right)\right] \cos ^{2} \alpha}{\exp \left(1 / 2 i \omega_{*}\right)-\exp \left(1 / 2 i \omega_{*} \cos ^{2} \alpha\right)}
\end{aligned}
$$

This link corresponds to a transfer function $\mathcal{H}$ in the frequency space which depends on the dimensionless operating flame conditions parameter $\Gamma$, the flame aspect ratio or flame angle $\alpha$ and the reduced angular frequency $\omega *=\omega R /\left(S_{L} \cos \alpha\right)$.

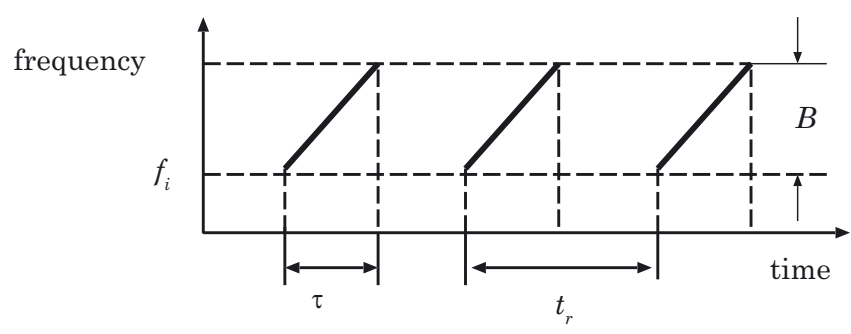

Fig. 3. Schematic representation of a train of "chirps".

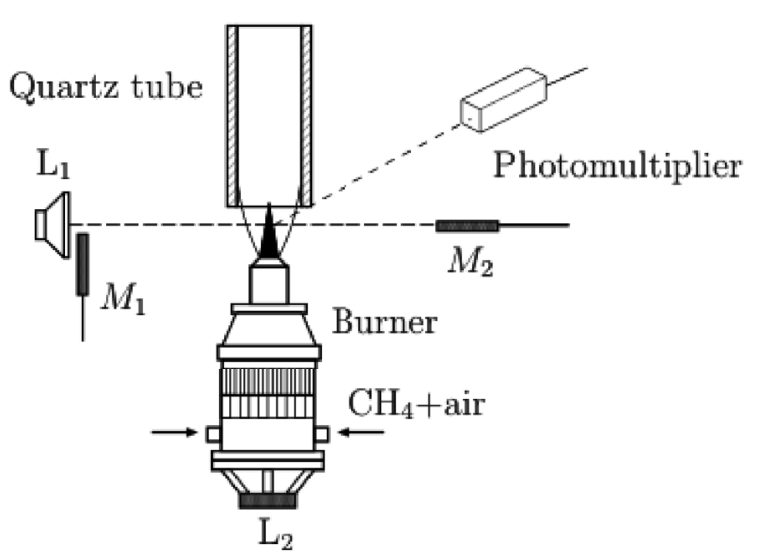

Fig. 4. Schematic view of the experimental setup.

\section{Experimental configuration}

Measurements are obtained on an axisymmetric burner with a $20 \mathrm{~mm}$ outlet nozzle diameter. Conical premixed flames can be stabilized on the lips of burner and are submitted to velocity modulations using a loudspeaker $L_{2}$ at the base of the burner. A train of waves, illustrated in Figure 3, is generated by a signal synthesizer, amplified and transmitted to the space by a tweeter $L_{1}$ placed on one side of the burner at the same height. The ultrasonic sound waves produced are recorded by two microphones $M_{1}$ and $\mathrm{M}_{2}, \mathrm{~B} \& \mathrm{~K}$ 4938, before and after their passages through the flame. The two microphones are well aligned to ensure that the acoustic path crosses through the axis of the burner. These signals are then digitized, amplified, high pass filtered and post-processed to extract the cross-correlation between the incident and transmitted sound waves. Each train of pulses is a chirp signal with a constant amplitude $\beta$ but with a Linear Frequency Modulation (LFM) over a certain frequency bandwidth $B$ and time duration $\tau$ (Fig. 3). This technique enables to obtain a narrow compressed pulse response for the cross-correlation between the incident and transmitted signals with a main peak corresponding to the sought sound travel time $\Delta t$. Provided that the repetition time $t_{r}$ between two successive chirps is large enough, the time resolution of the technique improves as $1 /(B \tau)$, where $B \tau$ is the compression factor [16]. The parameters of the chirp signal used in this study are $f_{i}=20 \mathrm{kHz}, B=20 \mathrm{kHz}$, $\tau=0.8 \mathrm{~ms}$ and $t_{r}=1.0 \mathrm{~ms}$. 


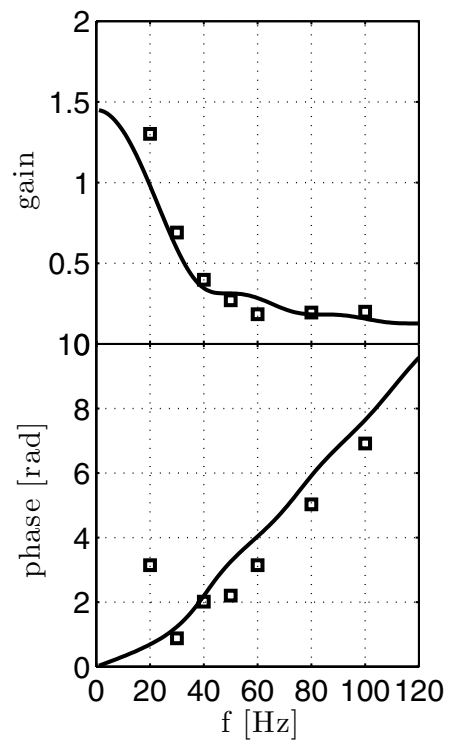

(a)

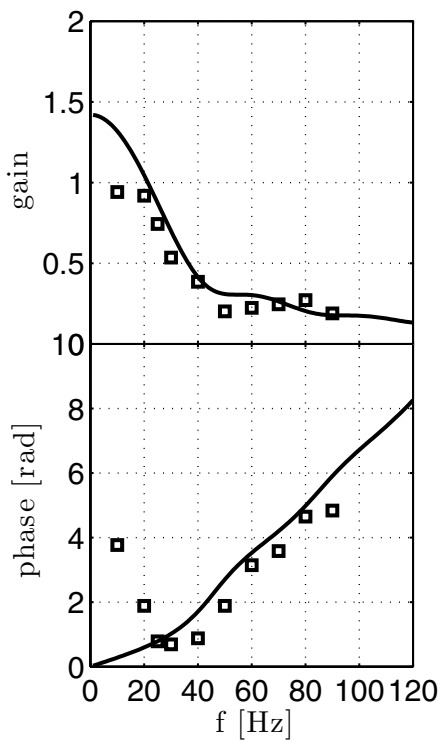

(b)

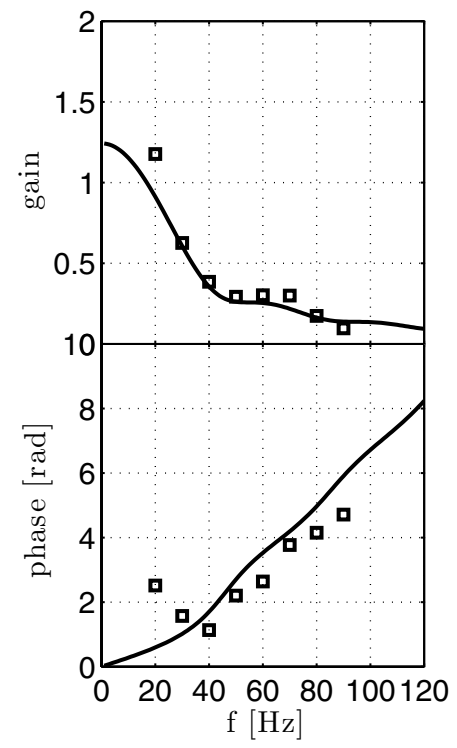

(c)

Fig. 5. Comparisons between analytical predictions and experimental results for the transfer function $\mathcal{H}$ as function of the modulation frequency $f$. Modulation level $v_{\mathrm{u}, \mathrm{rms}} / \bar{v}_{\mathrm{u}}=0.09$. (a) Flamme FL1 $\phi=0.9, \bar{v}_{\mathrm{u}}=1.5 \mathrm{~m} \cdot \mathrm{s}^{-1}$. (b) Flamme FL2 $\phi=1.0$, $\bar{v}_{\mathrm{u}}=1.5 \mathrm{~m} . \mathrm{s}^{-1}$. (c) Flamme FL3 $\phi=1.0, \bar{v}_{\mathrm{u}}=1.1 \mathrm{~m} . \mathrm{s}^{-1}$.

The flow velocity with and without modulation is measured by a hot-wire at the exist of the burner (in the absence of combustion). A quartz tube with a diameter of $50 \mathrm{~mm}$ and a length of $300 \mathrm{~mm}$ is used to partially confine the burned gases and limit buoyancy perturbing effects. A photomultiplier PM is also used to record the chemiluminescence emission from the flame. For well premixed flames in absence of mixture inhomogeneities, fluctuations of light intensity $I$ of the natural radicals $\mathrm{CH}^{*}, \mathrm{OH}^{*}$ or $\mathrm{C}_{2}^{*}$ present in the flame front are proportional to heat release rate perturbations [5] :

$$
\dot{Q}^{\prime} / \overline{\dot{Q}}=I^{\prime} / \bar{I}
$$

Using this optical signal and the acoustic measurements realized with the two microphones, it is possible to reconstruct the experimental transfer function $\mathcal{H}$ and compare the results with predictions from equation (10).

\section{Results}

Results are presented in Figure 5 for three methane/air flames at different equivalence ratios $\phi$ and flow velocities $\bar{v}_{\mathrm{u}}$ (corresponding to different flame heights $H):$ FL1, $\phi=0.9, \bar{v}_{\mathrm{u}}=1.5 \mathrm{~m} . \mathrm{s}^{-1}, H=42 \mathrm{~mm}, \mathrm{FL} 2$, $\phi=1.0, \bar{v}_{\mathrm{u}}=1.5 \mathrm{~m} . \mathrm{s}^{-1}, H=38 \mathrm{~mm}$ and FL3, $\phi=1.0$, $\bar{v}_{\mathrm{u}}=1.1 \mathrm{~m} \cdot \mathrm{s}^{-1}, H=26 \mathrm{~mm}$. The modulation frequency covers 20 to $100 \mathrm{~Hz}$ in the experiments and the modulation level is fixed to $v_{\mathrm{u}, \mathrm{rms}} / \bar{v}_{\mathrm{u}}=0.09$, where $v_{\mathrm{u}, \mathrm{rms}}$ is the root mean square value of the flow velocity measured at the burner outlet. A good agreement in the gain
$G=|\mathcal{H}|$ and the phase $\varphi=\arg (\mathcal{H})$ of the transfer function between predictions and measurements is found for all cases explored, except at low frequencies where buoyancy effects strongly disturb the acoustic signals.

\section{Conclusions}

First validations of an acoustic diagnostic to measure heat release rate perturbations were carried out on laminar conical premixed flames submitted to harmonic flow modulations. The results obtained are encouraging. A simple analytical model has been developed that can be used to test and validate various approximations to treat the acoustic signal and reconstruct fluctuations in heat release rate. Work is undergoing to validate the technique in more complex configurations.

Acknowledgements. Jingxuan Li is supported by a doctoral fellowship from China Scholarship Council, Project 111, Grant No. B08009. The authors wish to thank Dr. Daniel Durox for helpful discussion about buoyancy effects. Alexis Guquel, $\mathrm{Ph} . \mathrm{D}$. student at laboratory EM2C, is acknowledged for his help in checking the equations.

\section{References}

[1] S. Candel, Combustion dynamics and control: Progress and challenges, Proc. Comb. Inst. 29 (2002) 1-28

[2] A. Fayoux, K. Zahringer, O. Gicquel, J. Rolon, Experimental and numerical determination of heat release in counterflow premixed laminar flames, Proc. Comb. Inst. 30 (2005) 251-257 
[3] B. Ayoola, R. Balachandran, J. Frank, E. Mastorakos, C. Kaminski, Spatially resolved heat release rate measurements in turbulent premixed flames, Comb. Flame 144 (2006) 1-16

[4] I.R. Hurle, R.B. Price, T.M. Sugden, A. Thomas, Sound emission from open turbulent premixed flames, Royal Soc. London Proc. Ser. A 303 (1968) 409-427

[5] R. Price, I. Hurle, T. Sugden, Optical studies of the generation of noise in turbulent flames, Symp. (Int.) on Combustion 12 (1969) 1093-1102

[6] B. Higgins, M.Q. McQuay, F. Lacas, S. Candel, An experimental study on the effect of pressure and strain rate on $\mathrm{CH}$ chemiluminescence of premixed fuel-lean methane/air flames, Fuel 80 (2001) 1583-1591

[7] Y. Hardalupas, M. Orain, Local measurements of the time dependent heat release rate and equivalence ratio using chemiluminescent emission from a flame, Comb. Flame 139 (2004) 188-207

[8] M. Lauer, T. Sattelmayer, On the adequacy of chemiluminescence as a measure for heat release in turbulent flames with mixture gradients, J. Eng. Gas Turbine. Power 132 (2010) 1-8

[9] T. Lieuwen, Theory of high frequency acoustic wave scattering by turbulent flames, Comb. Flame 126 (2001) $1489-1505$
[10] T. Lieuwen, R. Rajaram, Y. Neumeier, S. Nair, Measurements of incoherent acoustic wave scattering from turbulent premixed flames, Proc. Comb. Inst. 29 (2002) 1809-1815

[11] G. Searby, Instability phenomena during flame propagation. In Combustion Phenomena: Selected mechanisms of flame formation, propagation, and extinction, in: J. Jarosinski and B. Veyssiere (ed.), CRC Press, New York, 2008, pp. 67-79

[12] M. Fleifil, A.M. Annaswamy, Z.A. Ghoneim, A.F. Ghoniem, Response of a laminar premixed flame to flow oscillations: A kinematic model and thermoacoustic instability results, Comb. Flame 106 (1996) 487-510

[13] T. Schuller, D. Durox, S. Candel, A unified model for the prediction of laminar flame transfer functions: comparisons between conical and $\mathrm{V}$ flame dynamics, Comb. Flame 134 (2003) 21-34

[14] S. Ducruix, D. Durox, S. Candel, Theoretical and experimental determinations of the transfer function of a laminar premixed flame, Proc. Comb. Inst. 28 (2000) 765-773

[15] F. Baillot, D. Durox, R. Prud'homme, Experimental and theoretical study of a premixed vibrating flame, Comb. Flame 88 (1992) 149-168

[16] S. Merrill, Radar Handbook, McGraw-Hill, New York, 2008 